Article

\title{
Development of a Class-C Power Amplifier with Diode Expander Architecture for Point-of-Care Ultrasound Systems
}

\author{
Hojong Choi \\ Department of Medical IT Convergence Engineering, Kumoh National Institute of Technology, Gumi 39253, \\ Korea; hojongch@kumoh.ac.kr; Tel.: +82-54-478-7782
}

Received: 8 September 2019; Accepted: 11 October 2019; Published: 14 October 2019

\begin{abstract}
Point-of-care ultrasound systems are widely used in ambulances and emergency rooms. However, the excessive heat generated from ultrasound transmitters has an impact on the implementation of piezoelectric transducer elements and on battery consumption, thereby affecting the system's sensitivity and resolution. Non-linear power amplifiers, such as class-C amplifiers, could substitute linear power amplifiers, such as class-A amplifiers, which are currently used in point-of-care ultrasound systems. However, class-C power amplifiers generate less output power, resulting in a reduction of system sensitivity. To overcome this issue, we propose a new diode expander architecture dedicated to power amplifiers to reduce the effects of sinusoidal pulses toward the power supply. Thus, the proposed architecture could increase the input pulse amplitudes applied to the main transistors in the power amplifiers, hence increasing the output voltage of such amplifiers. To verify the proposed concept, pulse-echo responses from an ultrasonic transducer were tested with the developed class- $C$ power amplifier using a resistor divider and the designed diode expander architecture. The peak-to-peak amplitude of the echo signals of the ultrasonic transducers when using a class-C power amplifier with a diode expander architecture $\left(2.98 \mathrm{~V}_{\mathrm{p}-\mathrm{p}}\right)$ was higher than that for the class-C power amplifier with a resistor divider architecture $\left(2.51 \mathrm{~V}_{\mathrm{p}-\mathrm{p}}\right)$. Therefore, the proposed class- $C$ power amplifier with diode expander architecture is a potential candidate for improving the sensitivity performance of piezoelectric transducers for point-of-care ultrasound systems.
\end{abstract}

Keywords: class-C power amplifier; diode expander; piezoelectric transducers; point-of-care ultrasound systems

\section{Introduction}

Ultrasound has been widely used for patient diagnosis because it is a non-ionizing, real-time, non-invasive, and inexpensive imaging modality [1]. Especially, point-of-care ultrasound systems have been used to diagnose acute diseases for immediate treatment in ambulance vehicles and emergency rooms with remote help from physicians [2,3]. However, the performance of point-of-care ultrasound systems typically suffers from a limited number of ultrasonic array transducer elements, overheating, and battery issues, thereby affecting the sensitivity and resolution of such ultrasound systems $[3,4]$. To overcome overheating and battery issues, manufacturers need to use cooling systems such as cooling fans and aluminum heat pipe structures even though the internal structures of point-of-care ultrasound systems are much smaller than conventional bench-top ultrasound machines [3].

Conventional ultrasound systems are composed of ultrasound transmitters, ultrasonic (piezoelectric) transducers, and ultrasound receivers [5-7]. Most of the overheating issues come from the power amplifiers in the ultrasound transmitters and by the analog-to-digital converter or digital-to-analog converter in the ultrasound receivers [8]. However, the excessive heat could be 
resolved by employing non-linear power amplifiers in the ultrasound transmitters owing to their different transition period between voltage and current [9]. Among non-linear power amplifiers, class-C power amplifiers could achieve relatively low DC current consumption while lowering the gain [10]. Therefore, the scheme to increase the gain would be beneficial for class-C power amplifiers in low-sensitivity ultrasonic transducers.

Several non-linear power amplifiers have been developed using different types of ultrasonic transducers to improve the performance of ultrasound instruments. A class-E inverter was proposed for inductive piezoelectric transducers to determine the optimal resonance frequency based on the equivalent circuit model [11]. A class-D half-bridge driving power amplifier was proposed for piezoelectric transducers to find the optimal conversion frequency operation [12]. A class-E power amplifier was proposed to reduce power dissipation in Langevin transducers [13].

Figure 1 describes how input signals in power amplifiers affect the bias circuit and how the power supply controls the power amplifiers through the bias circuit. Positive and negative pulse signals can pass through the bias circuit and get into the power supply while the power supply is applied to DC voltages through the bias circuit to the power amplifier [10]. In the output, the amplified positive and negative pulse signals trigger the ultrasonic transducer. The bias circuit needs to be stabilized during signal amplification through control of the power supply. Therefore, the pulse signals should not affect the bias circuit operation. Otherwise, it could generate output signals with high noise and harmonic distortion or reduce the output signal amplitudes. These scenarios directly affect the resolution and sensitivity performance of ultrasound systems [2,14].

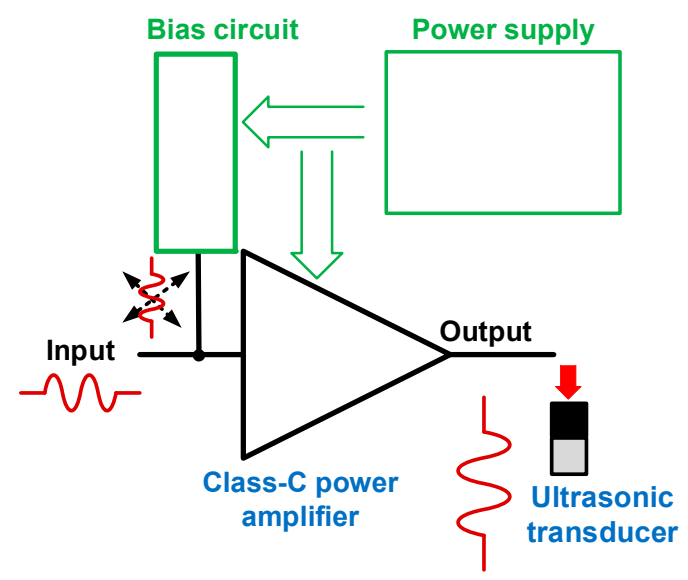

Figure 1. Concept for class-C power amplifiers with input pulse signals and bias circuit.

To reduce the effects of pulse signals on bias circuits, different architecture using an analog-to-digital converter, a digital-to-analog converter, and a look-up table have been used for wireless applications [15]. This method needs to regulate several DC bias voltages to properly operate the transistors. This might not be suitable for point-of-care ultrasound systems because of their higher DC current consumption. Additionally, a number of ultrasonic transducers are required for combination with each transmitter channel to achieve a limited size of point-of-care ultrasound systems. However, the proposed method is a much simpler approach for easy regulation of a single power amplifier, thereby increasing the output voltages of the power amplifiers for point-of-care ultrasound systems.7

A power metal-oxide semiconductor field-effect transistor (MOSFET) simulation model has been developed to estimate the expected performance in a high-voltage environment [9]. Given that a power MOSFET simulation library for a high-voltage environment provides inaccurate performance for sub-decibel level operation, the performance of power amplifiers needs to be verified through real measurements $[15,16]$. Therefore, in Section 2, we report the detailed experimental circuit design analysis in DC and AC levels rather than calculated and simulated results. The equivalent circuit model with measured parameters for power amplifiers is provided. Section 3 shows the measured 
performance results for the power amplifier including pulse-echo response with ultrasonic transducers. Section 4 concludes the paper.

\section{Materials and Methods}

Diode expanders placed after power amplifiers have originally been used to reduce unwanted ring down signals generated from class-A power amplifiers in ultrasound instruments. Class-A amplifiers produce a higher gain compared to class-C power amplifiers while consuming a higher DC current. Therefore, class-A power amplifiers can use relatively lower input voltage signals compared to class-C power amplifiers. In ultrasound instruments, the maximum allowable input voltages are generally limited to $1 \mathrm{~V}_{\mathrm{p}-\mathrm{p}}[17,18]$. Therefore, the bias circuits in class-A power amplifiers would be less affected by input pulse signals. However, class-C power amplifiers produce a relatively lower voltage gain compared to class-A power amplifiers and also consume a lower DC current. Consequently, higher input voltage signals could affect the bias circuit operation for class- $C$ power amplifiers. Overall, a diode expander architecture is proposed for bias circuits of class- $C$ power amplifiers because higher input pulse signals can pass through the bias circuits while the power supply controls the bias circuits and the amplified pulse signal reaches the ultrasonic transducers. The proposed diode expander architecture is intended to suppress unwanted input pulse signals into the bias circuits, resulting in stabilization of the DC current and improvement of the output voltages of class-C power amplifiers.

Figure 2 shows a schematic diagram and implemented printed circuit board of the developed class- $C$ power amplifier with a resistor divider and with a diode expander architecture. The printed circuit board was fabricated from a manufacturing service (ExpressPCB LLC, Mulino, OR, USA). The mounting holes were tied to reduce parasitic effects. The signal traces were not perpendicularly cross the ground plane. Given that high-voltage pulse signals are needed to trigger ultrasonic transducers, power film resistors, capacitors, inductors, choke inductors, and power transistors are usually selected because they tolerate high voltages. The selected power transistors ( $T_{1}, P_{D} 57018-E$, STMicroelectronics Inc., Geneva, Switzerland) were lateral-diffusion metal-oxide semiconductor field-effect transistors (LDMOSFETs) that can be used for high voltages up to $65 \mathrm{~V}$ and high currents up to 2.5 A operations. A small-size (1 cm length and width) heat-sink was attached on the top of the LDMOSFET devices. Electrolytic capacitors $\left(\mathrm{C}_{\mathrm{E} 1}=10 \mu \mathrm{F}\right.$ and $\left.\mathrm{C}_{\mathrm{E} 2}=220 \mu \mathrm{F}\right)$ including additional ceramic capacitors $\left(\mathrm{C}_{\mathrm{L} 1}=0.1 \mu \mathrm{F}, \mathrm{C}_{\mathrm{L} 2}=1 \mathrm{nF}\right.$, and $\left.\mathrm{C}_{\mathrm{L} 3}=47 \mathrm{pF}\right)$ admitting up to $200 \mathrm{~V}$ were used to stabilize the DC voltages from the power supplies. A radio frequency (RF) choke inductor with a self-resonant frequency at $190 \mathrm{MHz}\left(\mathrm{L}_{\mathrm{c}}=1 \mu \mathrm{H}\right)$ admitting high currents up to 2 A was selected. The power film resistors $\left(R_{1}\right.$ to $\left.R_{5}\right)$ operated up to $250 \mathrm{~V}$. The input and output matching circuits were used in the input and output sides, respectively, to match the operating frequency of the power amplifier. In the input matching circuit, one resistor, two capacitors, and one inductor were used. In the output matching circuit, three capacitors and two inductors were employed. In both matching circuits, the resistors, capacitors, and inductors also admitted high voltages, up to $100 \mathrm{~V}$. Cooling fans were used for accurate measurement, though class- $\mathrm{C}$ power amplifiers generate less heat than class- $\mathrm{A}$ power amplifiers. Table 1 provides component values for the power amplifiers.

Table 1. Component values for class-C power amplifiers.

\begin{tabular}{cccccccc}
\hline Components & Values & Components & Values & Components & Values & Components & Values \\
\hline $\mathrm{R}_{\mathrm{r} 1}$ & $5 \mathrm{k} \Omega$ & $\mathrm{C}_{\mathrm{E} 2}$ & $220 \mu \mathrm{F}$ & $\mathrm{C}_{5}$ & $150 \mathrm{pF}$ & $\mathrm{C}_{6}$ & $100 \mathrm{pF}$ \\
\hline $\mathrm{R}_{\mathrm{r} 2}$ & $0.5 \mathrm{k} \Omega$ & $\mathrm{C}_{\mathrm{L} 1}$ & $0.1 \mu \mathrm{F}$ & $\mathrm{C}_{\mathrm{L} 4}$ & $0.1 \mu \mathrm{F}$ & $\mathrm{L}_{\mathrm{c}}$ & $1 \mu \mathrm{H}$ \\
\hline $\mathrm{R}_{1}$ & $56 \Omega$ & $\mathrm{C}_{\mathrm{L} 2}$ & $1 \mathrm{nF}$ & $\mathrm{C}_{\mathrm{L} 5}$ & $1 \mathrm{nF}$ & $\mathrm{L}_{2}$ & $560 \mathrm{nH}$ \\
\hline $\mathrm{C}_{\mathrm{E} 1}$ & $10 \mu \mathrm{F}$ & $\mathrm{C}_{\mathrm{L} 3}$ & $47 \mathrm{pF}$ & $\mathrm{C}_{\mathrm{L} 6}$ & $47 \mathrm{pF}$ & $\mathrm{L}_{3}$ & $220 \mathrm{nH}$ \\
\hline
\end{tabular}




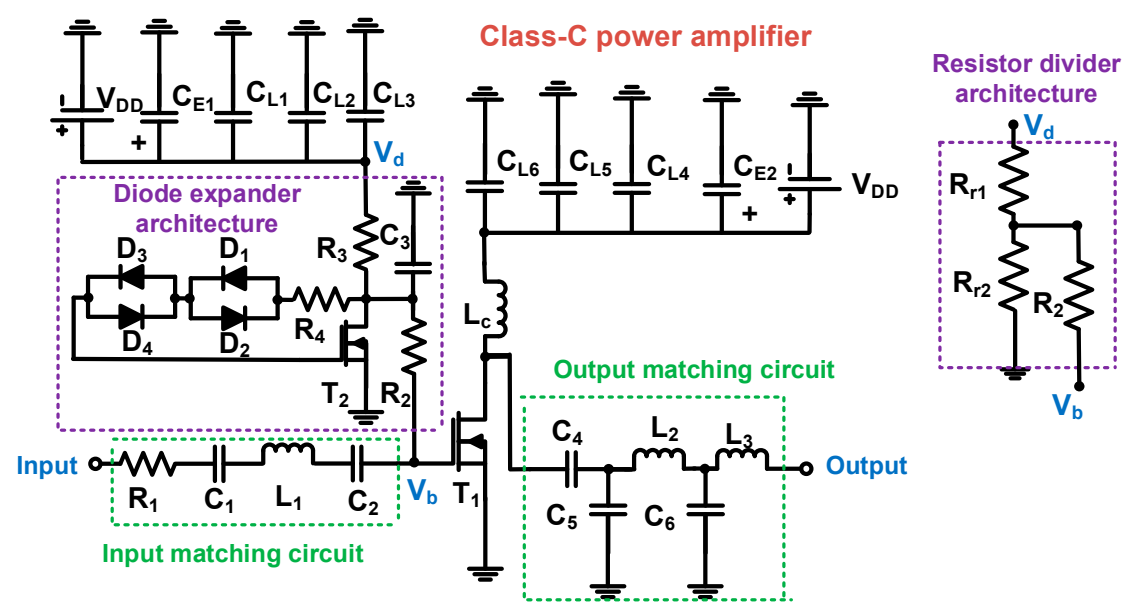

(a)

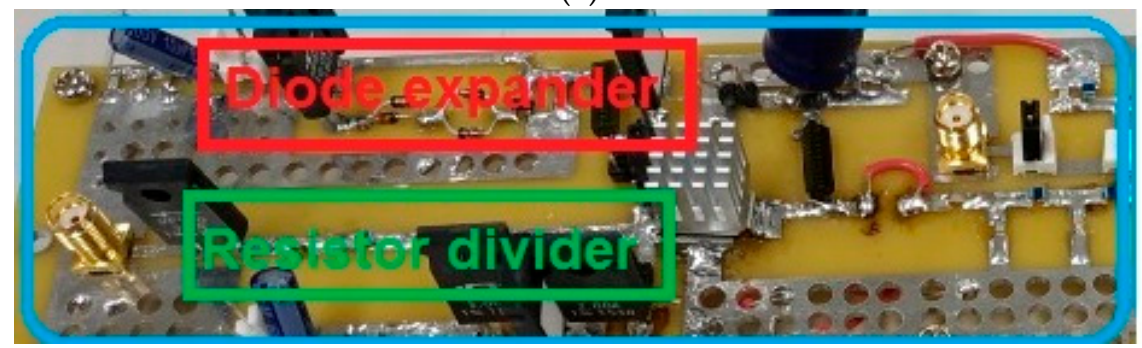

(b)

Figure 2. (a) Schematic diagram and (b) implemented printed circuit board of the developed class- $\mathrm{C}$ power amplifier with a resistor divider and diode expander architecture.

Figure 3 shows the operating mechanisms of the resistor divider and diode expander architecture including thermal resistances. Although the manufacturers did not provide the thermal model parameters of the power film resistors, diodes, and LDMOSFETs, their thermal resistances were included to estimate thermal effects in the resistor divider and diode expander architecture. In the resistor divide architecture, the DC bias voltages were obtained by the ratio of two power film resistors $\left(R_{r 1}=5 \mathrm{k} \Omega\right.$ and $R_{r 2}=500 \Omega$ ) with their corresponding thermal resistances $\left(R_{r 1 J C}\right.$ and $\left.R_{r 2 J C}\right)$. In the diode expander architecture, the DC bias voltages were obtained by two power film resistors, diode equivalent resistance $\left(R_{D}\right)$, and drain-source resistance of one of the LDMOSFET $\left(T_{2}\right)$ including their thermal resistances $\left(\mathrm{R}_{3 J \mathrm{C}}, \mathrm{R}_{4 \mathrm{JC}}, \mathrm{R}_{\mathrm{DJC}}\right.$, and $\left.\mathrm{R}_{\mathrm{T} 2 \mathrm{JC}}\right)$.

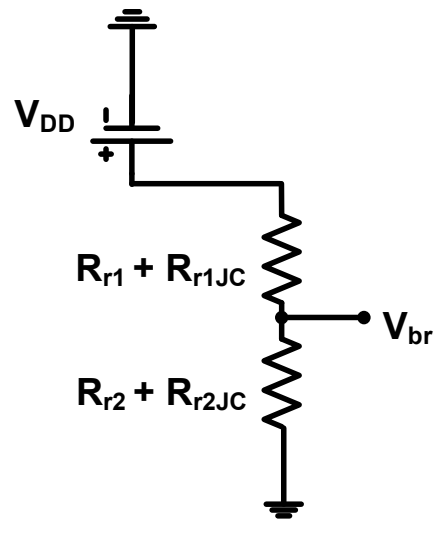

(a)

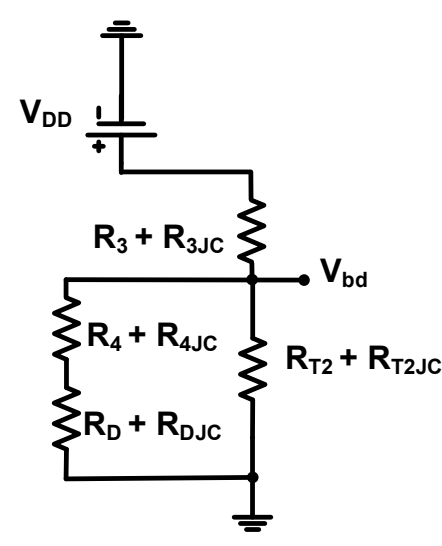

(b)

Figure 3. DC Operating mechanisms of the (a) resistor divider and (b) diode expander architecture. 
In Figure 3a, the resistor divider architecture used two power film resistors $\left(R_{r 1}\right.$ and $\left.R_{r 2}\right)$. The bias voltage of the resistor divider $\left(\mathrm{V}_{\mathrm{br}}\right)$ is expressed as:

$$
\mathrm{V}_{\mathrm{br}}=\frac{\mathrm{R}_{\mathrm{r} 2}+\mathrm{R}_{\mathrm{r} 2 J \mathrm{C}}}{\mathrm{R}_{\mathrm{r} 1}+\mathrm{R}_{\mathrm{r} 1 J \mathrm{C}}+\mathrm{R}_{\mathrm{r} 2}+\mathrm{R}_{\mathrm{r} 2 J \mathrm{C}}} \mathrm{V}_{\mathrm{dd}}=\frac{1}{1+\frac{\mathrm{R}_{\mathrm{r} 1}+\mathrm{R}_{\mathrm{r} 1 J \mathrm{C}}}{\mathrm{R}_{\mathrm{r} 2}+\mathrm{R}_{\mathrm{r} 2 \mathrm{C}}}} \mathrm{V}_{\mathrm{dd}}
$$

Here, $V_{d d}$ is the supply voltage from the DC power supply and $R_{r 1 J C}, R_{r 2 J C}$, and $R_{r 3 J C}$ are the thermal resistances of the power film resistors $\left(R_{r 1}, R_{r 2}\right.$, and $R_{r 3}$, respectively).

The power MOSFET equivalent circuit model has been used to estimate the performance of diode expander architecture $[19,20]$. The diode expander architecture in Figure 3b used double cross-coupled diodes. The bias voltage of this architecture $\left(\mathrm{V}_{\text {bias2 }}\right)$ is expressed as:

$$
\mathrm{V}_{\mathrm{bd}}=\frac{1}{1+\frac{\mathrm{R}_{3}+\mathrm{R}_{3 J \mathrm{C}}}{\left(\mathrm{R}_{\mathrm{T} 2}+\mathrm{R}_{\mathrm{T} 2 \mathrm{~J} \mathrm{C}}\right) / /\left(\mathrm{R}_{4}+\mathrm{R}_{4 J \mathrm{C}}+\mathrm{R}_{\mathrm{D}}+\mathrm{R}_{\mathrm{DJC}}\right)}} \mathrm{V}_{\mathrm{dd}} \approx \frac{1}{1+\frac{\mathrm{R}_{3}+\mathrm{R}_{3 J \mathrm{C}}}{\mathrm{R}_{\mathrm{T} 2}+\mathrm{R}_{\mathrm{T} 2 \mathrm{C}}}} \mathrm{V}_{\mathrm{dd}}
$$

where $R_{3 J C}, R_{T 2 J C}, R_{4 J C}$, and $R_{D J C}$ are the thermal resistances of the power film resistors $\left(R_{3}\right.$ and $\left.R_{4}\right)$, the diode equivalent resistance $\left(\mathrm{R}_{\mathrm{D}}\right)$, and the drain-source resistance of LDMOSFET $\mathrm{T}_{2}$.

The thermal effect on the bias voltages for the resistor divider architecture is dependent on the two power film resistors $\left(R_{\mathrm{r} 1}\right.$ and $\left.R_{\mathrm{r} 2}\right)$ with their corresponding thermal resistances $\left(R_{\mathrm{r} 1 \mathrm{JC}}\right.$ and $\left.\mathrm{R}_{\mathrm{r} 2 \mathrm{JC}}\right)$. Conversely, the thermal effect on the bias voltages for the diode expander architecture is dependent on a single power film resistor $\left(R_{3}\right)$ and the drain-source resistance of LDMOSFET R $R_{T 2}$, with their corresponding thermal resistances $\left(\mathrm{R}_{3 \mathrm{JC}}\right.$ and $\left.\mathrm{R}_{\mathrm{T} 2 \mathrm{JC}}\right)$. Therefore, the drain-source resistance of LDMOSFET $\mathrm{R}_{\mathrm{T} 2}$ is less insensitive to sudden temperature changes compared to power film resistors because temperature changes are dependent on resistance values [21]. To confirm these temperature dependences, the bias voltages for each architecture were measured after $1 \mathrm{~min}$ and $1 \mathrm{hr}$. At $1 \mathrm{~min}$, the measured bias voltage of the class- $C$ power amplifier when using the resistor divider and diode expander architecture was the same, i.e., $2.3 \mathrm{~V}$. After $1 \mathrm{~h}$, the measured bias voltage of the class-C power amplifier with the resistor divider and diode expander architecture did not coincide anymore; they were $2.25 \mathrm{~V}$ and $2.29 \mathrm{~V}$, respectively. Therefore, we confirm that class-C power amplifiers with diode expander architecture are less dependent on temperature variations.

Figure 4 shows the AC operating mechanisms to predict the expected input signal paths of class-C power amplifiers with the resistor divider and diode expander architecture. As shown in Figure 4a, the pulse signals from the input port (path 1) could be transmitted to two power film resistors ( $R_{r 1}$ and $R_{r 2}$, paths 3 and 4 ) through one power film resistor $\left(R_{2}\right)$. Likewise, Figure $4 b$ shows that the pulse signals from the same input port (path 1 ) could be split into one capacitor $\left(C_{3}\right.$, path 3 ), one power film resistor with cross-coupled diode pairs $\left(R_{4}, D_{1}\right.$ to $D_{4}$, path 4$)$, and one LDMOSFET $\left(T_{2}\right.$, path 5$)$. We estimated that there are more paths to alleviate the burden into the DC power caused by unwanted AC signals. Therefore, we expect that more AC input signals could get to LDMOSFET $\mathrm{T}_{1}$ for a class-C power amplifier with a diode expander architecture. To confirm this idea, the input voltages at the gate of the LDMOSFET $\left(\mathrm{V}_{\mathrm{b}}\right)$ were measured as depicted in Figure $4 \mathrm{c}$. The arbitrary function generator produced $5 \mathrm{~V}_{\mathrm{p}-\mathrm{p}}$ pulsed signals at $25 \mathrm{MHz}$ for class-C power amplifiers with the resistor divider and diode expander architecture using a 10:1 voltage probe (TPP0200, Tecktronics Inc., Beaverton, OR, USA) from a digital oscilloscope (MDO4104C, Tecktronics Inc., Santa Clara, CA, USA). The measured bias voltages of the class-C power amplifier with resistor divider and diode expander architecture were $0.21 \mathrm{~V}$ and $0.24 \mathrm{~V}_{\mathrm{p}-\mathrm{p}}$, respectively. Therefore, we can confirm that the class-C power amplifier with a diode expander architecture would be more suitable to reduce the voltage attenuation, resulting in higher output voltage amplification. 


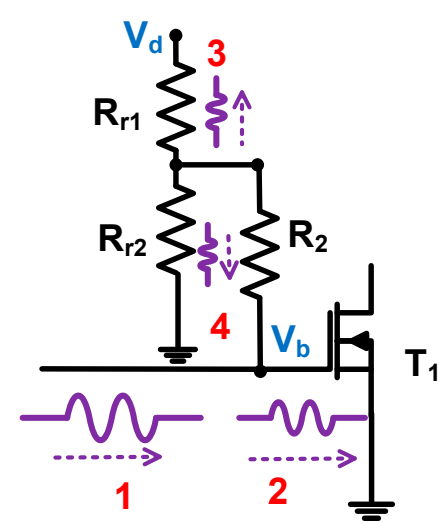

(a)

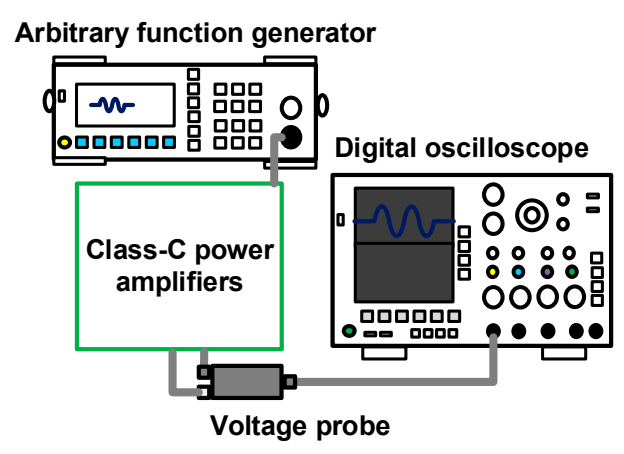

(c)

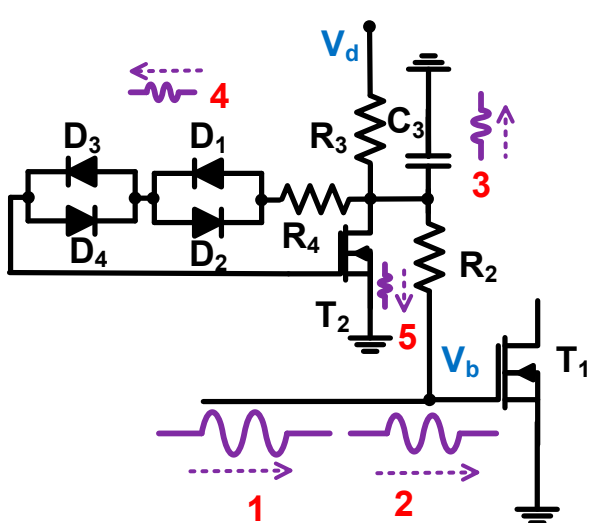

(b)

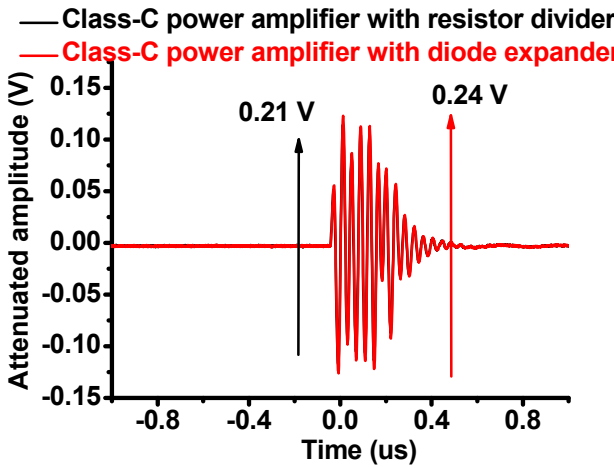

(d)

Figure 4. The AC operating mechanisms of the (a) resistor divider and (b) diode expander architecture; (c) measurement setup for input voltage attenuation in the class-C power amplifier; and the (d) measured $\mathrm{AC}$ input voltages at the gate of the LDMOSFET $\mathrm{T}_{1}$.

The gain $\left(\mathrm{V}_{\mathrm{G}}\right)$ and $\mathrm{DC}$ current consumption $\left(\mathrm{P}_{\mathrm{D}}\right)$ of the class-C power amplifiers can be expressed $[9,22]$ as:

$$
\begin{gathered}
\mathrm{V}_{G}=20 \cdot \log _{10}\left(\mathrm{i}_{\mathrm{o}} \cdot \frac{\mathrm{R}_{\mathrm{L}}}{2 \pi \cdot \mathrm{V}_{\mathrm{in}}}\left(2 \theta_{c}-\sin 2 \theta_{c}\right)\right) \\
\mathrm{I}_{\mathrm{D}}=\frac{\mathrm{i}_{\mathrm{o}}}{\pi}\left(\sin \theta_{c}-\theta_{c} \cos \theta_{c}\right)
\end{gathered}
$$

Here, $i_{0}, R_{L}, V_{i n}$, and $\theta_{c}$ are the output current, load resistance, input voltage, and conduction angle of the class- $C$ power amplifiers.

The gain and DC current consumption equations for class-C power amplifiers with resistor divider and diode expander architecture coincide [22]. However, the output current $\left(\mathrm{i}_{\mathrm{o}}\right)$ and conduction angles $\left(\theta_{c}\right)$ are different given that those values are dependent on the DC bias voltages [22]. The LDMOSFET $\mathrm{T}_{1}$ cannot provide the expected parameter for low DC bias voltage in class-C-type power amplifiers. There are no simulation model parameters either for power film resistors (CADDOCK Electronics Inc., Roseburg, OR, USA) and LDMOSFET $\mathrm{T}_{2}$ in the diode expander. Therefore, modeled equations of the gain and DC current consumption are provided without simulation data. The measured performance of the class-C power amplifier with resistor divider and diode expander architecture is characterized next.

\section{Results and Discussion}

To estimate the performance of the class-C power amplifier with a resistor divider and diode expander architecture, the measurement setup shown in Figure 5a for voltage gain versus input voltage 
of power amplifiers was employed. A $25 \mathrm{MHz}$ five-cycle sinusoidal pulse signal with up to $5 \mathrm{~V}_{\mathrm{p}-\mathrm{p}}$ was generated from an arbitrary function generator (DG5701, Rigol Inc., Beijing, China) and applied to the designed class-C power amplifiers to measure the output peak-to-peak voltage amplitude in a digital oscilloscope (MDO4104C). One DC power supply (2231A-3-30, Keithley Instruments, Cleveland, OH, USA) provided gate-source DC voltage and another power supply (E3647A, Agilent Technologies, Santa Clara, CA, USA) provided drain-source DC voltage for the class-C power amplifiers. The voltage gain in each case was obtained by dividing the measured output peak-to-peak voltage by the corresponding measured input peak-to-peak voltage. The pulse-echo mode is a standard method to evaluate the performance of the developed electronic components or ultrasonic transducers [23]. In the pulse-echo mode, the voltage amplitude of the echo signal generated by ultrasonic transducers is typically measured in terms of peak-to-peak voltage [24]. Therefore, voltage gain was measured through peak-to-peak voltages for ultrasound systems.

Figure $5 b, c$ show the measured voltage gain versus input voltages of a class- $C$ power amplifier with resistor divider and diode expander architecture. The voltage gain of the amplifier with diode expander architecture $(14.96 \mathrm{~dB})$ was higher than that of the amplifier with resistor architecture $(12.04 \mathrm{~dB})$ at $5 \mathrm{~V}_{\mathrm{p}-\mathrm{p}}$ input. Additionally, the gain deviation of the amplifier with diode expander architecture $(16.17 \mathrm{~dB})$ was lower than that of the amplifier with resistor architecture $(24.43 \mathrm{~dB})$ at $5 \mathrm{~V}_{\mathrm{p}-\mathrm{p}}$ input. Therefore, the class- $C$ power amplifier with a diode expander architecture exhibited higher voltage gain compared to that with a resistor divider architecture. Figure $5 \mathrm{e}, \mathrm{f}$ show the measured voltage gain versus frequencies of the class- $C$ power amplifier with the resistor divider and diode expander architecture. The voltage gain of the class- $C$ power amplifier with the diode expander architecture $(1.81 \mathrm{~dB})$ was higher than that of the amplifier with the resistor architecture $(-52.39 \mathrm{~dB})$ at $50 \mathrm{MHz}$. Additionally, gain deviation of the amplifier with diode expander architecture $(-0.38 \mathrm{~dB})$ is lower than that of the amplifier with resistor architecture $(-20.69 \mathrm{~dB})$ at $50 \mathrm{MHz}$. Therefore, the class-C power amplifier with the diode expander architecture exhibited a relatively wider bandwidth compared to that with the resistor divider architecture.

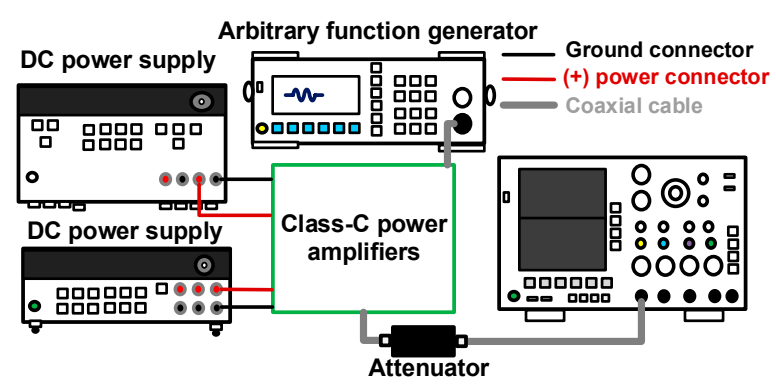

(a)

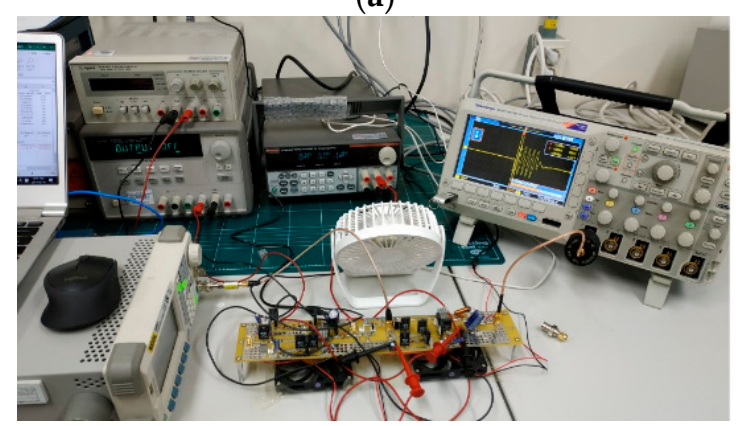

(b)

Figure 5. Cont. 


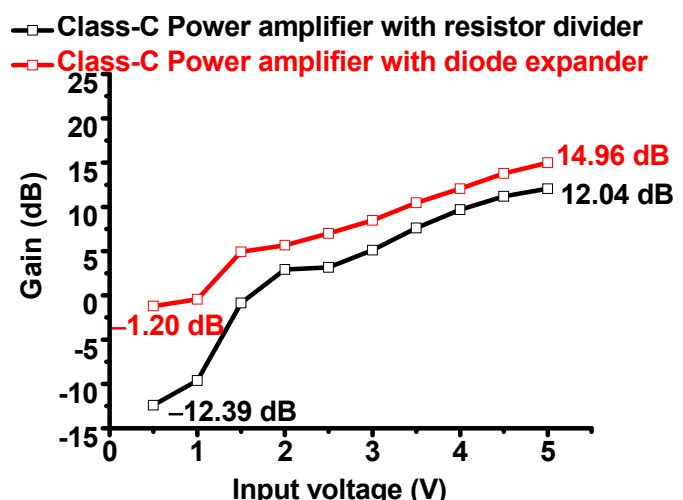

(c)

- - Class-C Power amplifier with resistor divider - Class-C Power amplifier with diode expander

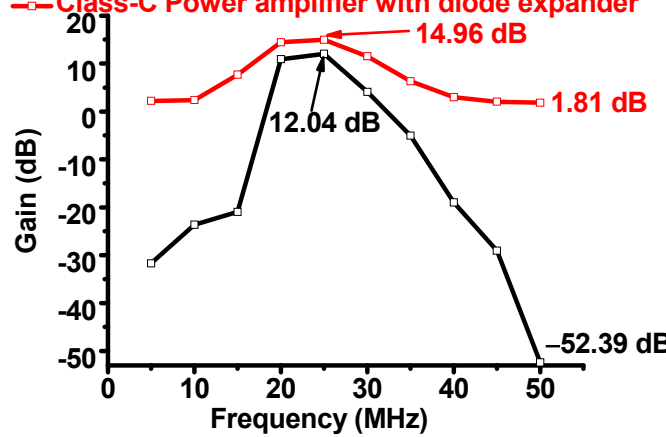

(e)

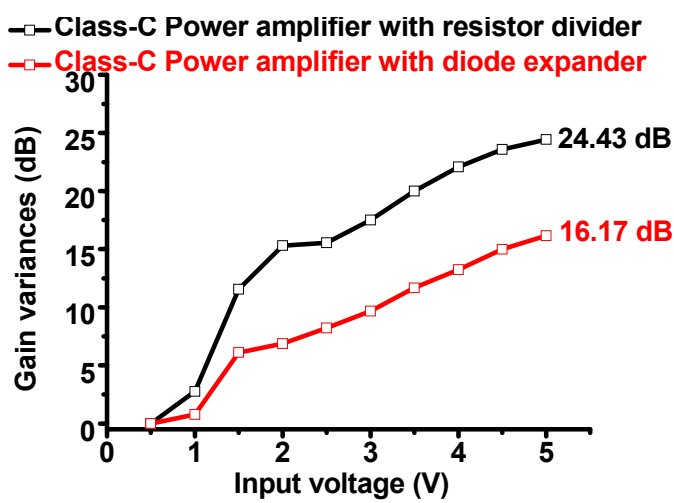

(d)

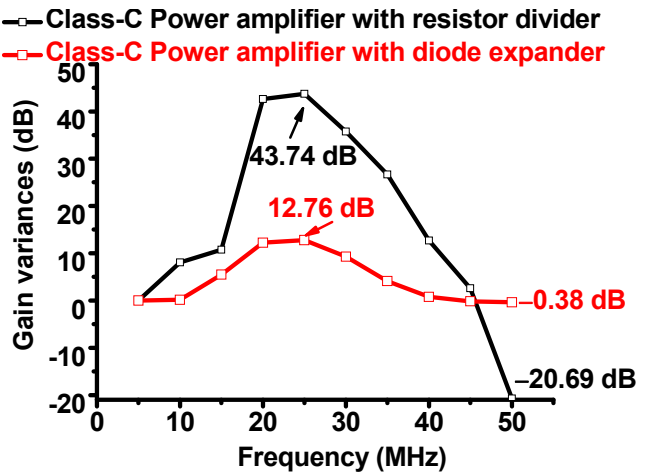

(f)

Figure 5. (a) Measurement setup sketch and (b) an image of its actual realization for measurement of voltage gain and DC current consumption of the power amplifiers; measured voltage gain of the class-C power amplifier versus input voltage with the (c) resistor divider and diode expander architecture; (d) measured voltage gain deviation of the class-C power amplifier versus input voltage with resistor divider and diode expander architecture; measured voltage gain of the class-C power amplifier versus frequency with the (e) resistor divider and (f) diode expander architecture.

Measured data for DC current consumption versus input voltages and frequencies of the class-C power amplifiers with the resistor divider and diode expander architecture are depicted in Figure $6 a, b$. In Figure 6a, a $25 \mathrm{MHz}$ sinusoidal pulse signal with up to $5 \mathrm{~V}_{\mathrm{p}-\mathrm{p}}$ was applied to the designed class-C power amplifier to measure the current consumption. In Figure $6 \mathrm{~b}$, a $5 \mathrm{~V}_{\mathrm{p}-\mathrm{p}}$ sinusoidal pulse signal up to $50 \mathrm{MHz}$ was applied to the designed class-C power amplifiers to measure the current consumption. The measured current consumption of the class-C power amplifier with resistor diode and diode expander architecture was less than $1 \mathrm{~A}$. The measured current consumption of the amplifier with the diode expander architecture (0.52 A) was a bit higher than that of the amplifier with the resistor divider architecture $(0.36 \mathrm{~A})$. Therefore, both class-C power amplifiers still generated low current consumption such that it can produce relatively low heat, resulting in lower battery consumption for point-of-care ultrasound systems.

The currently designed class- $C$ power amplifier with the diode expander architecture had a reasonable gain between $15 \mathrm{MHz}$ and $35 \mathrm{MHz}$. To re-design the amplifier to cover the bandwidth between $1 \mathrm{MHz}$ and $25 \mathrm{MHz}$, the input and output matching circuits need to be removed and series capacitors may be used. For such a case, the bandwidth between $1 \mathrm{MHz}$ and $25 \mathrm{MHz}$ could be covered in the class- $C$ power amplifiers. However, the voltage gain could be decreased or increased as the frequency increased. To flat the bandwidth, the linearizer circuit would be helpful [25]. Therefore, the diode expander architecture needs to be replaced with another linearizer circuit. Therefore, each architecture has some trade-offs in terms of performance, such as voltage gain and bandwidth. 


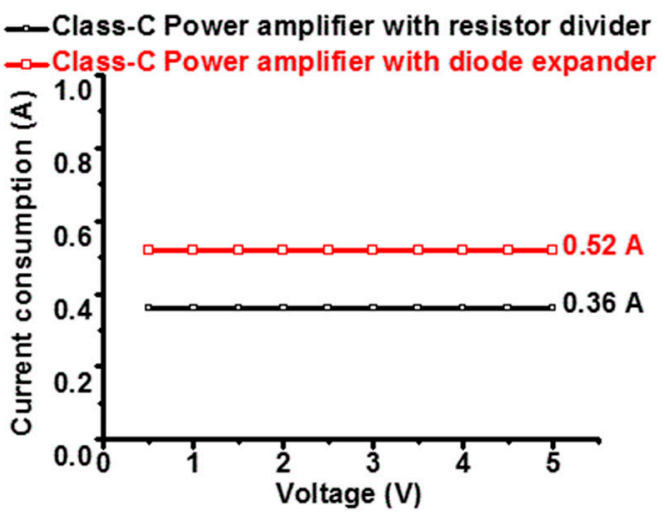

(a)

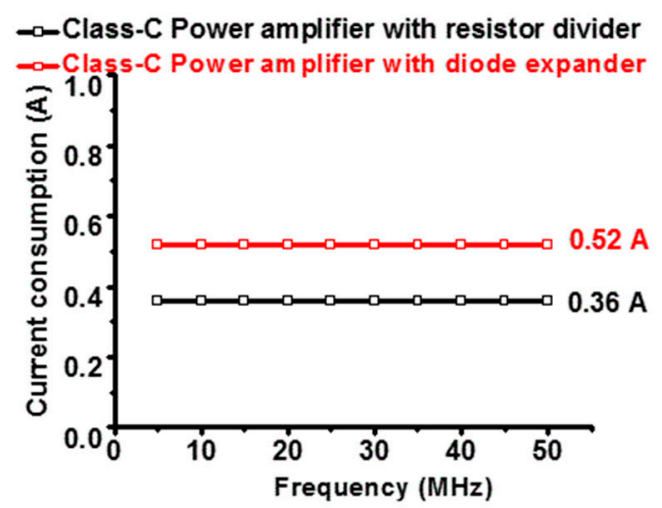

(b)

Figure 6. (a) Current consumptions of the class-C power amplifier versus input voltage and (b) frequency with the resistor divider and diode expander architecture.

There is more research that needs to be done for the use of higher frequencies for a variety of applications, such as skin, eye, and intravascular imaging $[23,26]$. The future direction of point-of-care ultrasound systems could utilize a frequency range higher than $25 \mathrm{MHz}$, since current commercial bench-top ultrasound machines are already doing so [2]. Higher frequency operation in ultrasound machines will be more demanding due to the higher spatial resolution while scarifying the penetration depth [2].

Figure 7a shows a typical setup for pulse-echo response measurement using class-C power amplifiers with a resistor divider and diode expander architecture [18]. A $25 \mathrm{MHz}$ five-cycle $5 \mathrm{~V}_{\mathrm{p}-\mathrm{p}}$ sinusoidal waveform from a generator (DG5701) was the input of the designed class-C power amplifiers. This input triggered the ultrasonic transducer (V324, Olympus Scientific Solutions Americas Inc., Waltham, MA, USA). Ultrasonic transducers transmitted acoustic waveforms into a circular quartz target. The reflected weak acoustic waveforms were converted into electrical waveforms by ultrasonic transducers. The resulting waveforms were then amplified by a $36 \mathrm{~dB}$ preamplifier (AU-1526, L3 Narda-MITEQ Inc., Hauppauge, NY, USA) to be displayed on a $1 \mathrm{GHz}$ digital oscilloscope with a 5 GS/s sampling rate (MDO3102). Figure 7b,c show echo signal amplitudes and their normalized spectrum when using the class-C power amplifier with a resistor divider and diode expander architecture. The echo signal amplitude is related with the sensitivity of ultrasonic transducers [27-29]. In Figure 7b, echo signal amplitudes when using the class-C power amplifiers with resistor divider $\left(2.51 \mathrm{~V}_{\mathrm{p}-\mathrm{p}}\right)$ is a little bit smaller than that when using the amplifiers with diode expander architecture $\left(2.98 \mathrm{~V}_{\mathrm{p}-\mathrm{p}}\right)$. The $-6 \mathrm{~dB}$ bandwidth is related with the image resolution of ultrasonic transducers in point-of-care ultrasound systems [30-32]. In Figure 7c, the echo signal spectrum when using class-C power amplifiers with a resistor divider (17.51\%) was smaller than that when using the amplifiers with a diode expander architecture (18.52\%). Therefore, the class-C power amplifier with a diode expander architecture outperformed the class-C power amplifier with a resistor divider architecture when it came to improving the sensitivity of point-of-care ultrasound systems. 


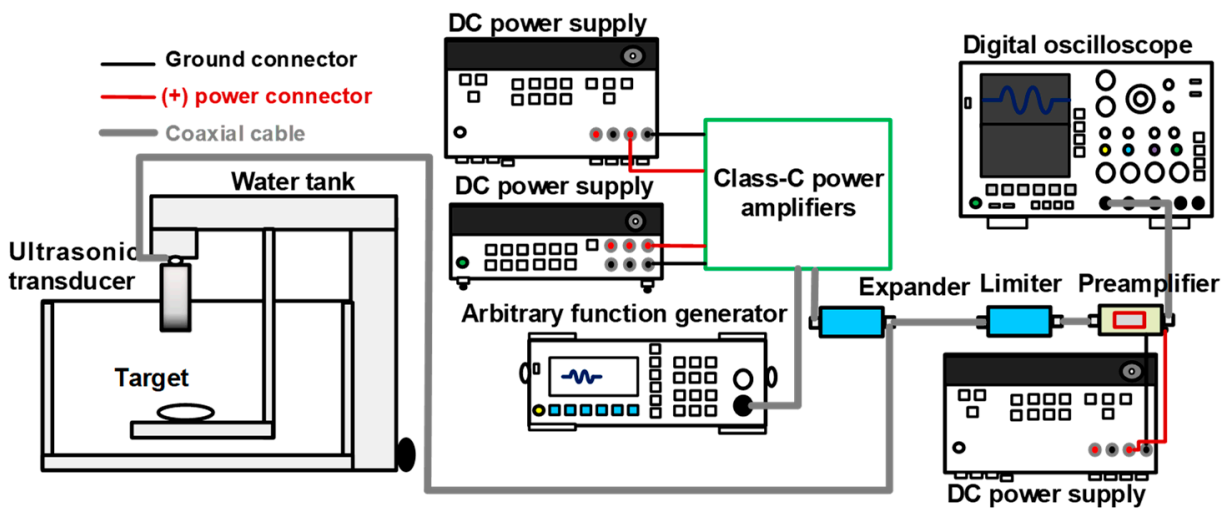

(a)

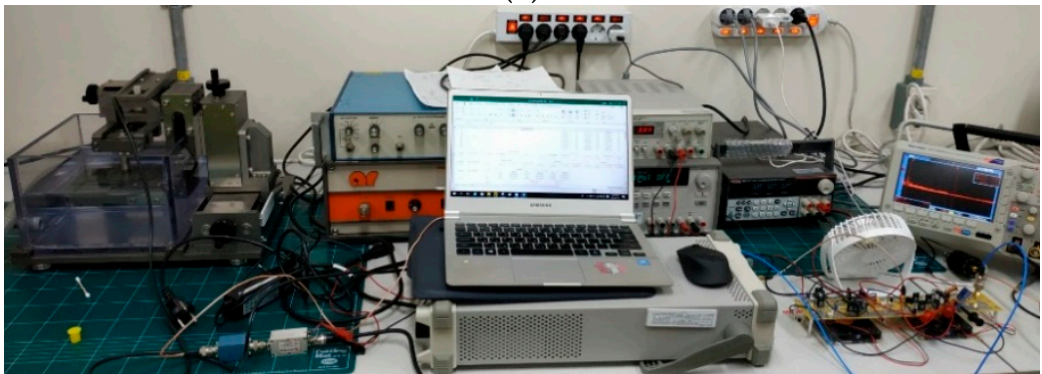

(b)

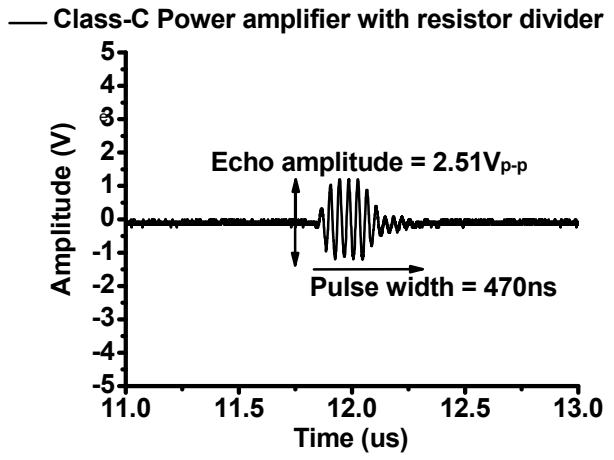

(c)

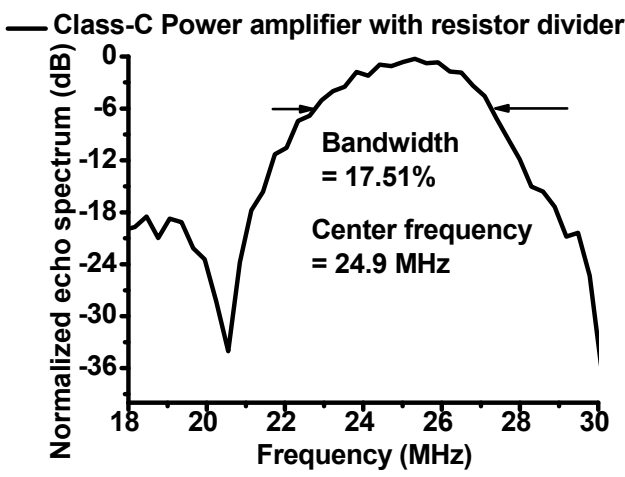

(e)

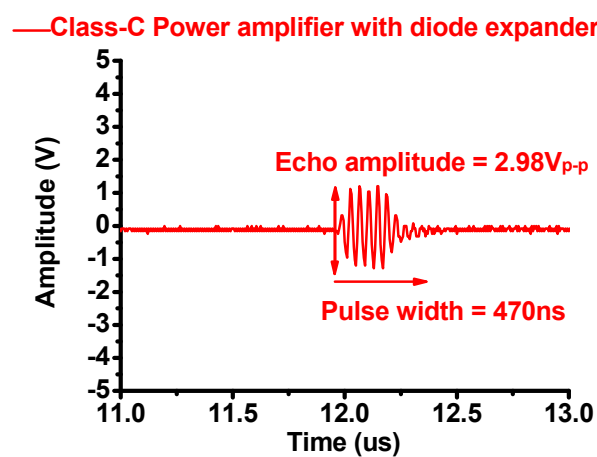

(d)

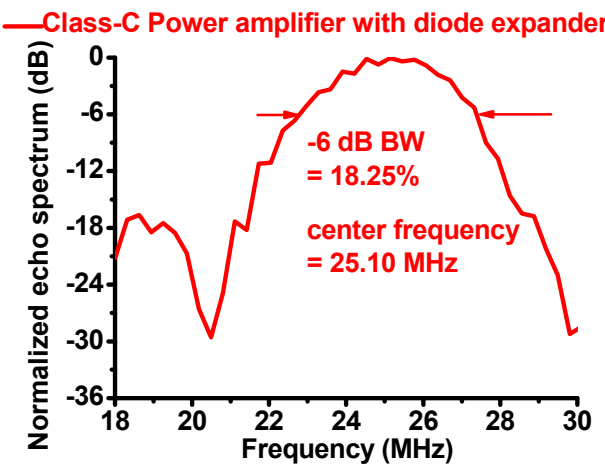

(f)

Figure 7. (a) Schematic diagram and (b) pictures during pulse-echo response measurement; echo signal amplitudes when using the class-C power amplifier with a (c) resistor divider and (d) diode expander architecture; echo signal spectrums when using the class-C power amplifier with a (e) resistor divider and (f) diode expander architecture. 


\section{Conclusions}

In point-of-care ultrasound systems, excessive overheating critically reduces the performance of piezoelectric transducers because class-A power amplifiers in ultrasound transmitters generate unwanted heat during the entire operation. To reduce excessive overheating, non-linear power amplifiers such as class- $C$ power amplifiers can be used given that these amplifiers have on and off transition periods during its entire operation. Because of pulse signals affecting bias circuits, class-C power amplifiers could be affected by bias circuits, resulting in the generation of sensitive outputs for ultrasonic transducers. Therefore, a new diode expander architecture dedicated to improving input signal conditions for class-C power amplifiers was proposed. The diode expander architecture could reduce the effects of unwanted input pulse signals toward bias circuits, thus reducing the attenuation of the input pulse signals for class- $C$ power amplifiers. As a result, higher input signals could be transferred to the class- $C$ power amplifiers. We have shown that the gain of a class- $C$ power amplifier with a diode expander $(14.96 \mathrm{~dB})$ was higher than that with a resistor diode expander $(12.04 \mathrm{~dB})$ for a 5 $\mathrm{V}_{\mathrm{p}-\mathrm{p}}$ input. However, the current consumption of a class- $\mathrm{C}$ power amplifier with a diode expander architecture $(1.02 \mathrm{~W})$ was a little bit higher than that with a resistor divider architecture $(0.75 \mathrm{~W})$.

To confirm the proposed idea, typical one-way pulse-echo response measurements were taken. The echo signal amplitude and its $-6 \mathrm{~dB}$ bandwidth when using a class- $C$ power amplifier with diode expander architecture (2.98 $\mathrm{V}_{\mathrm{p}-\mathrm{p}}$ and $\left.18.25 \%\right)$ was higher and wider than those of a class-C power amplifier with resistor divider architecture $\left(2.51 \mathrm{~V}_{\mathrm{p}-\mathrm{p}}\right.$ and $\left.17.51 \%\right)$. The limitation of the developed class-C power amplifier with a resistor divider architecture made it difficult to block the unwanted pulse input signals. The limitation of the developed class- $C$ power amplifier with a diode expander architecture is that the maximum voltage of the LDMOSFET in the diode expander needs to be much higher than the power supply with unwanted pulse signals. However, a class-C power amplifier with diode expander architecture can be a useful way to improve the output voltage amplitude. In the future, the developed architecture combined with a multiplexer/de-multiplexer will be applied to the array transducers because the sensitivity in point-of-care ultrasound systems is one of the critical performance issues.

Funding: This research was supported by the Basic Science Research Program through the National Research Foundation of Korea (NRF) funded by the Ministry of Science, ICT and Future Planning (NRF-2017R1C1B1003606).

Conflicts of Interest: The author declares no conflict of interest.

\section{References}

1. Shung, K.K. Diagnostic Ultrasound: Imaging and Blood Flow Measurements; Taylor Francis: Boca Raton, FL, USA, 2015.

2. Szabo, T.L. Diagnostic Ultrasound Imaging: Inside Out; Elsevier Academic Press: London, UK, 2013.

3. Daniels, J.M.; Hoppmann, R.A. Practical Point-of-Care Medical Ultrasound; Springer: New York, NY, USA, 2016.

4. Choi, H.; Choe, S.W. Therapeutic Effect Enhancement by Dual-bias High-voltage Circuit of Transmit Amplifier for Immersion Ultrasound Transducer Applications. Sensors 2018, 18, 4210. [CrossRef] [PubMed]

5. Choi, H. Prelinearized Class-B Power Amplifier for Piezoelectric Transducers and Portable Ultrasound Systems. Sensors 2019, 19, 287. [CrossRef] [PubMed]

6. Shin, S.-H.; Yoo, W.-S.; Choi, H. Development of Public Key Cryptographic Algorithm Using Matrix Pattern for Tele-Ultrasound Applications. Mathematics 2019, 7, 752. [CrossRef]

7. Zhu, B.; Fei, C.; Wang, C.; Zhu, Y.; Yang, X.; Zheng, H.; Zhou, Q.; Shung, K.K. Self-focused AlScN film ultrasound transducer for individual cell manipulation. ACS Sens. 2017, 2, 172-177. [CrossRef] [PubMed]

8. Choi, H.; Yoon, C.; Yeom, J.-Y. A Wideband High-Voltage Power Amplifier Post-Linearizer for Medical Ultrasound Transducers. Appl. Sci. 2017, 7, 354. [CrossRef]

9. Kazimierczuk, M.K. RF Power Amplifier; John Wiley Sons: Hoboken, NJ, USA, 2014.

10. Albulet, M. RF Power Amplifiers; SciTech Publishing: London, UK, 2001. 
11. Yuan, T.; Dong, X.; Shekhani, H.; Li, C.; Maida, Y.; Tou, T.; Uchino, K. Driving an inductive piezoelectric transducer with class E inverter. Sens. Actuators A 2017, 261, 219-227. [CrossRef]

12. Mortimer, B.; Du Bruyn, T.; Davies, J.; Tapson, J. High power resonant tracking amplifier using admittance locking. Ultrasonics 2001, 39, 257-261. [CrossRef]

13. Dong, X.; Yuan, T.; Hu, M.; Shekhani, H.; Maida, Y.; Tou, T.; Uchino, K. Driving frequency optimization of a piezoelectric transducer and the power supply development. Rev. Sci. Instrum. 2016, 87, 105003. [CrossRef]

14. Choi, H.; Park, C.; Kim, J.; Jung, H. Bias-Voltage Stabilizer for HVHF Amplifiers in VHF Pulse-Echo Measurement Systems. Sensors 2017, 17, 2425. [CrossRef]

15. Kenington, P.B. High Linearity RF Amplifier Design; Artech House, Inc.: Norwood, MA, USA, 2000.

16. Vuolevi, J.; Rahkonen, T. Distortion in RF Power Amplifiers; Artech House: London, UK, 2003.

17. Choi, H.; Woo, P.C.; Yeom, J.-Y.; Yoon, C. Power MOSFET Linearizer of a High-Voltage Power Amplifier for High-Frequency Pulse-Echo Instrumentation. Sensors 2017, 17, 764. [CrossRef]

18. Jeong, J.J.; Choi, H. An impedance measurement system for piezoelectric array element transducers. Measurement 2017, 97, 138-144. [CrossRef]

19. Minasian, R.A. Power MOSFET dynamic large-signal model. IEE Proc. I Solid-State Electron Device 1983, 130, 73-79. [CrossRef]

20. Rohde, U.L.; Rudolph, M. RF/Microwave Circuit Design for Wireless Applications; John Wiley Sons: Hoboken, NJ, USA, 2013.

21. Razavi, B. RF Microelectronics; Prentice Hall: Upper Saddel River, NJ, USA, 2011.

22. Lee, T.H. The Design of CMOS Radio-Frequency Integrated Circuits; Cambridge University Press: Cambridge, UK, 2006.

23. Zhou, Q.; Lam, K.H.; Zheng, H.; Qiu, W.; Shung, K.K. Piezoelectric single crystal ultrasonic transducers for biomedical applications. Prog. Mater. Sci. 2014, 66, 87-111. [CrossRef] [PubMed]

24. Choi, H.; Yeom, J.Y.; Ryu, J.M. Development of a Multiwavelength Visible-Range-Supported Opto-Ultrasound Instrument Using a Light-Emitting Diode and Ultrasound Transducer. Sensors 2018, 18, 3324. [CrossRef] [PubMed]

25. Choi, H.; Jung, H.; Shung, K.K. Power Amplifier Linearizer for High Frequency Medical Ultrasound Applications. J. Med. Biol. Eng. 2015, 35, 226-235. [CrossRef] [PubMed]

26. Wang, X.; Seetohul, V.; Chen, R.; Zhang, Z.; Qian, M.; Shi, Z.; Yang, G.; Mu, P.; Wang, C.; Huang, Z. Development of a mechanical scanning device with high-frequency ultrasound transducer for ultrasonic capsule endoscopy. IEEE Trans. Med. Imaging 2017, 36, 1922-1929. [CrossRef] [PubMed]

27. Choi, H.; Choe, S.W. Acoustic Stimulation by Shunt-Diode Pre-Linearizer Using Very High Frequency Piezoelectric Transducer for Cancer Therapeutics. Sensors 2019, 19, 357. [CrossRef] [PubMed]

28. Qiao, Y.; Zhang, X.; Zhang, G. Acoustic radiation force on a fluid cylindrical particle immersed in water near an impedance boundary. J. Acoust. Soc. Am. 2017, 141, 4633-4641. [CrossRef]

29. Choi, H.; Ryu, J.; Kim, J. A Novel Fisheye-Lens-Based Photoacoustic System. Sensors 2016, 16, 2185. [CrossRef]

30. Choi, H. Class-C Linearized Amplifier for Portable Ultrasound Instruments. Sensors 2019, 19, 898. [CrossRef]

31. Choe, S.W.; Choi, H. Suppression Technique of HeLa Cell Proliferation Using Ultrasonic Power Amplifiers Integrated with a Series-Diode Linearizer. Sensors 2018, 18, 4248. [CrossRef] [PubMed]

32. Choi, H.; Ryu, J.M.; Yeom, J.Y. Development of a Double-Gauss Lens Based Setup for Optoacoustic Applications. Sensors 2017, 17, 496. [CrossRef] [PubMed]

(C) 2019 by the author. Licensee MDPI, Basel, Switzerland. This article is an open access article distributed under the terms and conditions of the Creative Commons Attribution (CC BY) license (http://creativecommons.org/licenses/by/4.0/). 